\section{Ciprofloxacin dry powder for inhalation in non-cystic fibrosis bronchiectasis: a phase II randomised study}

\author{
Robert Wilson*, Tobias Welte*, Eva Polverino", Anthony De Soyza ${ }^{+}$, Hugh Greville ${ }^{\S}$, \\ Anne O’Donnell ${ }^{f}$, Jeff Alder**, Peter Reimnitz ${ }^{\# \#}$ and Barbara Hampel ${ }^{4 \uparrow}$
}

ABSTRACT: This phase II, randomised, double-blind, multicentre study (NCT00930982) investigated the safety and efficacy of ciprofloxacin dry powder for inhalation (DPI) in patients with non-cystic fibrosis bronchiectasis.

Adults who were culture positive for pre-defined potential respiratory pathogens (including Pseudomonas aeruginosa and Haemophilus influenzae) were randomised to ciprofloxacin DPI $\mathbf{3 2 . 5} \mathbf{~ m g}$ or placebo administered twice daily for $\mathbf{2 8}$ days (with $\mathbf{5 6}$ days of follow-up). Bacterial density in sputum (primary end-point), pulmonary function tests, health-related quality of life and safety were monitored throughout the study.

60 subjects received ciprofloxacin DPI $32.5 \mathrm{mg}$ and 64 received placebo. Subjects on ciprofloxacin DPI had a significant reduction $(p<0.001)$ in total sputum bacterial load at the end of treatment (-3.62 $\log _{10} \mathrm{CFU} \cdot \mathrm{g}^{-1}$ (range $\left.-9.78-5.02 \log _{10} \mathrm{CFU} \cdot \mathrm{g}^{-1}\right)$ ) compared with placebo $\left(-0.27 \log _{10} \mathrm{CFU} \cdot \mathrm{g}^{-1}\right.$ (range $\left.\left.-7.96-5.25 \log _{10} \mathrm{CFU} \cdot \mathrm{g}^{-1}\right)\right)$; the counts increased thereafter. In the ciprofloxacin DPI group, 14 (35\%) out of 40 subjects reported pathogen eradication at end of treatment versus four $(8 \%)$ out of 49 in the placebo group $(p=0.001)$. No abnormal safety results were reported and rates of bronchospasm were low.

Ciprofloxacin DPI 32.5 mg twice daily for 28 days was well tolerated and achieved significant reductions in total bacterial load compared with placebo in subjects with non-cystic fibrosis bronchiectasis.

KEYWORDS: Antibiotic, bacteria, chronic lung infection, exacerbation, inflammation, Pseudomonas aeruginosa

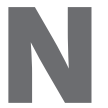
on-cystic fibrosis (CF) bronchiectasis is a chronic and debilitating respiratory disease that severely affects the healthrelated quality of life (HRQoL) of patients. The disease is characterised by abnormal and irreversible bronchial dilation, and is associated with increased morbidity and mortality attributable to airway obstruction, poor gas transfer and chronic bronchial infection [1-3]. Up to $64 \%$ of patients with bronchiectasis are chronically infected with pathogenic bacteria, most commonly Haemophilus influenzae and Pseudomonas aeruginosa [3]. These infections damage host defences and the respiratory epithelium, and can result in a vicious cycle of infection and inflammation with persistent symptoms, frequent acute exacerbations and disease progression [4]. One of the main therapeutic challenges is the clinical management of chronic airway infection because of frequent antibiotic resistance (especially to oral antibacterials), common side-effects of systemically administered antibacterials and intolerance to inhaled antibacterials (bronchospasm). These problems make non-CF bronchiectasis a difficult disease to treat and there is currently no approved antibacterial therapy for long-term treatment [3-6].

Off-label treatments for non-CF bronchiectasis include systemic and nebulised antibacterials, oral and inhaled mucolytics, hyperosmolar agents, bronchodilators and sputum clearance techniques $[7,8]$. However, these treatments do not adequately manage the number of exacerbations that patients may experience and, in some cases, their benefits have not been investigated fully. The lack of adequate evidence supporting
AFFILIATIONS

*Host Defence Unit, Royal Brompton Hospital, London, and ${ }^{+}$Newcastle University and Freeman Hospital, Newcastle upon Tyne, UK. \#Hannover Medical School,

Hannover,

\#\#Bayer Pharma AG, Wuppertal, and "Bayer Pharma AG, Berlin, Germany.

-Hospital Clinic de Barcelona IDIBAPS, CIBERES, Barcelona, Spain. ${ }^{\S}$ Royal Adelaide Hospital, Adelaide, Australia.

${ }^{f}$ Georgetown University Hospital, Washington, DC, and **Bayer HealthCare Pharmaceuticals Inc., Montville, NJ, USA.

CORRESPONDENCE

R. Wilson

Royal Brompton Hospital

London SW3 6NP

UK

E-mail: r.wilson@rbht.nhs.uk

Received:

May 042012

Accepted after revision:

Sept 152012

First published online:

Sept 272012

This article was modified in April 2016 to correct errors in the licence information. 
their use for managing exacerbations of bronchiectasis is noted in current European guidelines [9]. There is a need to develop new therapies that will effectively treat the underlying chronic infection, thereby reducing bronchial suppuration and the number of exacerbations, and preventing disease progression.

Ciprofloxacin, a broad-spectrum antibacterial, has proven bactericidal activity against pathogens relevant to non-CF bronchiectasis, including $P$. aeruginosa [10]. Ciprofloxacin has been formulated into a dry powder for inhalation (DPI) using PulmoSphere ${ }^{\mathrm{TM}}$ technology (Novartis Pharma AG, Basel, Switzerland) for use with the small, portable, breath-actuated T-326 inhaler [11]. Controlling the particle size and density of the active drug using PulmoSphere technology allows for optimal drug delivery to the central and lower airways. Results from clinical trials conducted in healthy volunteers and patients with CF and chronic obstructive pulmonary disease showed that ciprofloxacin DPI was well tolerated, with pharmacokinetics reflecting efficient lung targeting and low systemic exposure [12-14].

In addition to measures of respiratory function, techniques to assess health status are increasingly being evaluated and have been validated in patients with non-CF bronchiectasis. These include the St George's Respiratory Questionnaire (SGRQ), a self-completed questionnaire devised to measure HRQoL in individuals with chronic airflow limitation $[15,16]$. In addition, the Fatigue Impact Scale has been used to measure the correlation between fatigue and markers of disease severity [17].

The aim of this study was to assess the safety and efficacy of ciprofloxacin DPI treatment for 28 days, with 56 days of follow-up, in individuals with non-CF bronchiectasis by examining changes in bacterial load and other important clinical outcomes, as well as tolerability.

\section{MATERIALS AND METHODS Study subjects}

Adult subjects aged $\geqslant 18$ years were eligible for inclusion into the study if they had: either proven and documented diagnosis of idiopathic or a post-infective non-CF bronchiectasis (documentation of investigations to exclude other aetiologies reviewed) confirmed by high-resolution computerised tomography (radiology report reviewed); a history of exacerbations (two or more courses of systemic antibacterials or one or more hospitalisation for intravenous antibacterial treatment for pulmonary exacerbations in the previous 12 months); stable pulmonary status, defined as stable symptoms not requiring a new therapeutic intervention in the preceding 30 days; stable disease for $\geqslant 30$ days before study entry; and if they were able to produce a pre-treatment sputum sample of $\geqslant 5 \mathrm{~mL}$ that was culture positive for a pre-defined potential respiratory pathogen (P. aeruginosa (mucoid or nonmucoid), Staphylococcus aureus, Streptococcus pneumoniae, H. influenzae, Moraxella catarrhalis, Enterobacteriaceae, Stenotrophomonas maltophilia and Achromobacter xylosoxidans). Further details are included in the online supplementary material.

Study exclusion criteria included: active nontuberculous mycobacterial infection, recent significant haemoptysis (a volume requiring clinical intervention, within the previous 4 weeks) and the use of nebulised antibacterials as maintenance treatment, or systemic antibacterials for an exacerbation, within the 4 weeks before randomisation. Subjects on long-term antibacterials could stop for 1 month and then enter the study. To concede to clinical practice, chronic macrolide use was allowed as a stable anti-inflammatory maintenance therapy for bronchiectasis, but only if therapy had been commenced for at least the last 30 days before randomisation.

Subjects who had an exacerbation or another indication requiring administration of an antibiotic were discontinued from the study.

The conduct of this study met all local legal and regulatory requirements and was in accordance with the ethical principles of the Declaration of Helsinki and the International Conference on Harmonisation guideline E6: Good Clinical Practice. Written informed consent was provided by all subjects.

\section{Study design}

This was a multicentre, randomised, placebo-controlled, double-blind study (registered at www.clinicaltrials.gov with identifier number NCT00930982). Enrolled subjects received either ciprofloxacin DPI $32.5 \mathrm{mg}$ (corresponding to $50 \mathrm{mg}$ dry powder) or matching placebo b.i.d., dispensed from a T-326 inhaler (Novartis). Subjects were screened up to 21 days prior to day 1 and received treatment for 28 days, with follow-up visits at the end of treatment (EOT), and days 42, 56 and 84 (fig. 1). Study treatments were administered after standard maintenance therapy.

Pulmonary function tests were conducted on day 1 before the first treatment administration, $30 \pm 10 \mathrm{~min}$ after first treatment

\begin{tabular}{|c|c|}
\hline $\begin{array}{c}\text { Screening } \\
\text { Within } 21 \text { days before day } 1 \text {, visit } 1 \\
\text { Subjects enrolled }(n=277)\end{array}$ & \multirow{3}{*}{\begin{tabular}{|l} 
Subjects enrolled but \\
not randomised $(n=153)$ \\
Adverse event $(n=13)$ \\
Consent withdrawn $(n=12)$ \\
Lost to follow-up $(n=6)$ \\
Protocol violations $(n=122)$
\end{tabular}} \\
\hline$\nabla$ & \\
\hline $\begin{array}{c}\text { Baseline procedures and randomisation } \\
\text { Day 1, visit } 2 \\
\text { Subjects randomised }(n=124)\end{array}$ & \\
\hline $\begin{array}{c}\text { Ciprofloxacin DPI } 32.5 \mathrm{mg} \text { b.i.d days } 1-28(\mathrm{n}=60) \\
\text { Placebo b.i.d on days } 1-28(\mathrm{n}=64)\end{array}$ & \multirow{3}{*}{\begin{tabular}{|l} 
Analysis populations \\
Modified intent-to-treat \\
$(n=124)$ \\
Per-protocol $(n=82)$ \\
Safety $(n=124)$
\end{tabular}} \\
\hline$\downarrow$ & \\
\hline $\begin{array}{l}\text { Treatment visit } \\
\text { Day } 8 \pm 1 \text {, visit } 3\end{array}$ & \\
\hline \\
\hline \multicolumn{2}{|l|}{\begin{tabular}{|c|} 
End of treatment visit \\
Day $28 \pm 1$, visit 4 \\
Subjects completed study drug treatment $(n=103)$ \\
\end{tabular}} \\
\hline$\downarrow$ & \\
\hline \multicolumn{2}{|l|}{$\begin{array}{l}\text { 2-week follow-up } \\
\text { Day } 42 \pm 2 \text {, visit } 5\end{array}$} \\
\hline \multicolumn{2}{|l|}{$\downarrow$} \\
\hline 4-week follow-up & \\
\hline Day $56 \pm 2$, visit 6 & \multirow{4}{*}{$\begin{array}{l}\text { Premature termination }(n=50) \\
\text { Protocol violation }(n=6) \\
\text { Consent withdrawn }(n=2) \\
\text { Adverse events }(n=42) \\
\text { Exacerbations }(n=29) \\
\text { Other infections }(n=6) \\
\text { Bronchospasm }(n=3) \\
\text { Other }(n=4)\end{array}$} \\
\hline$\downarrow$ & \\
\hline $\begin{array}{l}\text { 8-week follow-up/end of study visit } \\
\text { Day } 84 \pm 2, \text { visit } 7 \\
\text { Subjects completed study }(n=74)\end{array}$ & \\
\hline & \\
\hline
\end{tabular}

FIGURE 1. Study design and subject disposition. Protocol violations in screening failures included no positive culture $(n=23)$, inadequate sputum $(n=50)$, forced expiratory volume in 1 s outside range $(n=6)$, exacerbations during screening $(n=2)$, on antibacterials $(n=6)$, no further information $(n=25)$ and other $(n=10)$. DPI: dry powder for inhalation. 
administration, on days 8, 29, 42, 56 and 84, and on premature discontinuation from the study. Spontaneous sputum samples for cultures and susceptibility testing were collected at each study visit before administration of treatment and on premature discontinuation from the study. In addition, subjects collected 24-h sputum samples on the day before days 1, 8, 29, 42, 56 and 84 .

A patient-reported HRQoL measure, the SGRQ, was completed by subjects on days 1, 29, 56 and 84 and on premature discontinuation from the study.

The incidence of abnormal findings in physical examinations and laboratory test results and the occurrence of adverse events (AEs) were monitored at each visit from screening to day 84 or until premature discontinuation from the study. Significant bronchospasm (defined as $\geqslant 15 \%$ decrease in absolute forced expiratory volume (FEV1)), local irritation and coughing were AEs of special interest because of the inhalational administration of treatment. Vital sign parameters (blood pressure and pulse rate) were measured on days 1, 8, 29 and 84 and on premature discontinuation from the study.

\section{Interim analysis}

A planned interim analysis was conducted after the primary end-point was reached in $\sim 70$ subjects, assessed at EOT. The independent data monitoring committee advised the unaltered continuation of the study. Further details are included in the online supplementary material.

\section{Study end-points}

The primary efficacy end-point was to determine the effect of ciprofloxacin DPI on total bacterial density of pre-defined potential respiratory pathogens in sputum, by measuring the reduction in $\log _{10}$ CFUs per gram of sputum after the 28-day treatment period and comparing the difference between ciprofloxacin DPI and placebo in the modified intent-to-treat (mITT) population (defined in the online supplementary material).

Secondary end-points included time to exacerbation, emergence of new potential respiratory pathogens, emergence of resistance among baseline pathogens, changes in inflammatory biomarkers, and change in 24-h sputum volume and colour from baseline. Changes from baseline in pulmonary function (FEV1 and forced vital capacity (FVC)) and in health status (SGRQ) at days 29, 56 and 84 were also evaluated, in addition to AEs and results of physical examinations, vital signs and laboratory analyses. Study-defined "eradication" was failure of bacterial growth from a valid post-baseline sputum sample in subjects with a positive baseline culture (all subjects). Samples were taken at screening, randomisation, day 8 (during treatment), EOT, and 14, 28 and 56 days after EOT. Samples were also taken at premature discontinuation of the study.

\section{Statistical analyses}

Two populations were considered for efficacy analysis: the mITT and per-protocol (defined in the online supplementary material populations). The primary analysis was performed on both mITT and per-protocol populations and was a comparison of change in total bacterial load at EOT between ciprofloxacin DPI and placebo, using a two-way ANCOVA method with baseline bacterial load as covariate and factors including treatment group and study centre.
Changes in sputum colour and volume were analysed by colour charts and 24-h sputum volume was analysed by frequency tables. The principal investigator provided investigators with photographs of mucoid, yellow, green and brown sputum. Changes in FEV1 and FVC were analysed by a twoway ANCOVA with baseline values as covariates and with factors including treatment group and study centre. Lung function parameters (FEV1 and FVC) as absolute raw values and $\%$ predicted were analysed with descriptive statistics.

The safety analysis set (defined in the online supplementary material) was used for all safety assessments. AEs that occurred after the start of study treatment and up to 7 days after EOT were classed as treatment emergent or worsening of existing AEs. Laboratory variables, vital signs and other safety data were described by summary statistics (defined in the online supplementary material).

Sample size estimation is described in the online supplementary material.

\section{RESULTS}

\section{Study population}

In total, 277 subjects were screened and 124 subjects were randomised to treatment (103 completed treatment and 74 completed the study) at 35 centres in six countries (Australia, Germany, Spain, Sweden, the UK and the USA) from June 29, 2009 to September 17, 2010. Of the randomised subjects, 60 received ciprofloxacin DPI $32.5 \mathrm{mg}$ b.i.d. and 64 received placebo b.i.d. (fig. 1). Most subjects who discontinued before randomisation provided an inadequate sputum sample (i.e. a sample from which the pre-defined pathogens could not be cultured). All subjects randomised were included in the safety and mITT populations, and 82 subjects (37 and 45 in the ciprofloxacin DPI and placebo groups, respectively) were included in the per-protocol population. Despite the high number of subjects excluded from the per-protocol population (mainly because EOT cultures were not available due to subjects not producing sputum), the results of the efficacy analysis in both the mITT and per-protocol populations were similar. The mITT data for all end-points are presented here, as are the per-protocol results for the primary end-point. The remainder of the per-protocol results are presented in the online supplementary material.

Demographic and baseline characteristics were similar in both treatment groups (table 1). There were no significant differences between ciprofloxacin DPI and placebo for any of the baseline characteristics. Age, sex and race characteristics were similar to those of patients who were screened but not randomised.

\section{Efficacy}

Treatment compliance and duration

Treatment compliance, measured by counting the number of both used/unused capsules and used/unused devices, was high across both treatment groups; compliance of $\geqslant 80 \%$ was reached in $97 \%$ of subjects in the ciprofloxacin DPI group and $94 \%$ of subjects in the placebo group. The mean treatment duration was 26 days in both treatment groups. 


\begin{tabular}{|c|c|c|c|}
\hline \multirow{2}{*}{$\begin{array}{l}\text { TABLE } 1 \\
\text { Characteristic }\end{array}$} & \multicolumn{3}{|c|}{$\begin{array}{l}\text { Demographic and baseline data (modified } \\
\text { intent-to-treat/safety population) }\end{array}$} \\
\hline & & $\begin{array}{l}\text { Ciprofloxacin } \\
\text { DPI }\end{array}$ & Placebo \\
\hline \multicolumn{2}{|l|}{ Subjects $n$} & 60 & 64 \\
\hline \multicolumn{2}{|l|}{ Age years } & $64.7 \pm 11.8$ & $61.4 \pm 11.9$ \\
\hline \multicolumn{4}{|l|}{ Sex } \\
\hline \multicolumn{2}{|l|}{ Male } & $21(35.0)$ & $21(32.8)$ \\
\hline \multicolumn{2}{|l|}{ Female } & $39(65.0)$ & $43(67.2)$ \\
\hline \multicolumn{4}{|l|}{ Ethnicity } \\
\hline \multicolumn{2}{|l|}{ Caucasian } & $60(100.0)$ & $63(98.4)$ \\
\hline \multicolumn{2}{|l|}{ Asian } & 0 & $1(1.6)$ \\
\hline \multicolumn{2}{|l|}{ Weight kg } & $70.2 \pm 16.4$ & $69.1 \pm 17.2$ \\
\hline \multicolumn{2}{|l|}{ BMI $\mathbf{k g} \cdot \mathrm{m}^{-2}$} & $25.6 \pm 5.5$ & $25.1 \pm 5.6$ \\
\hline \multicolumn{2}{|l|}{ FEV $1 \%$ pred } & $57.2 \pm 13.7$ & $54.6 \pm 14.8$ \\
\hline \multicolumn{4}{|c|}{$\begin{array}{l}\text { Subjects with exacerbations in } \\
\text { previous } 12 \text { months }\end{array}$} \\
\hline \multicolumn{2}{|c|}{ 1-2 exacerbations } & $30(50.0)$ & $35(54.7)$ \\
\hline \multicolumn{2}{|c|}{ 3-4 exacerbations } & $25(41.7)$ & $26(40.6)$ \\
\hline \multicolumn{2}{|c|}{$\geqslant 5$ exacerbations } & $5(8.3)$ & $3(4.7)$ \\
\hline \multicolumn{2}{|c|}{$\begin{array}{l}\text { Baseline total bacterial load } \log _{10} \\
\text { CFU } \cdot g^{-1}\end{array}$} & $7.19 \pm 1.94$ & $6.92 \pm 1.90$ \\
\hline \multicolumn{4}{|c|}{$\begin{array}{l}\text { Baseline bacterial isolates in } \\
\quad \geqslant 5 \% \text { subjects }{ }^{\#}\end{array}$} \\
\hline \multicolumn{2}{|c|}{ Pseudomonas aeruginosa } & $32(53.3)$ & $35(54.7)$ \\
\hline \multicolumn{2}{|c|}{ Haemophilus influenzae } & $14(23.3)$ & $16(25.0)$ \\
\hline \multicolumn{2}{|c|}{ Staphylococcus aureus } & $8(13.3)$ & $17(26.6)$ \\
\hline \multicolumn{2}{|c|}{ Streptococcus pneumoniae } & $7(11.7)$ & $2(3.1)$ \\
\hline \multicolumn{2}{|c|}{ Moraxella catarrhalis } & $5(8.3)$ & $3(4.7)$ \\
\hline \multicolumn{2}{|c|}{ Klebsiella pneumoniae } & $5(8.3)$ & 0 \\
\hline \multicolumn{2}{|c|}{ Proteus mirabilis } & $3(5.0)$ & $4(6.3)$ \\
\hline \multicolumn{2}{|c|}{ Klebsiella oxytoca } & $3(5.0)$ & $2(3.1)$ \\
\hline \multicolumn{4}{|c|}{$\begin{array}{l}\text { Data are presented as mean } \pm \text { SD or } n(\%) \text {, unless otherwise stated. DPI: dry } \\
\text { powder for inhalation; BMI: body mass index; FEV } 1 \text { : forced expiratory volume in } \\
1 \mathrm{~s} ; \% \text { pred: \% predicted. }{ }^{\#} \text { : no significant differences between ciprofloxacin } \\
\text { DPI and placebo for isolates were reported apart from for } K \text {. pneumoniae } \\
(p=0.024) \text {. }\end{array}$} \\
\hline
\end{tabular}

\section{Primary end-point}

A significantly greater mean reduction in CFU count during treatment and at EOT was observed in the ciprofloxacin DPI group $\left(-3.62 \log _{10} \mathrm{CFU} \cdot \mathrm{g}^{-1}\right.$, range $\left.-9.78-5.02 \log _{10} \mathrm{CFU} \cdot \mathrm{g}^{-1}\right)$ versus the placebo group $\left(-0.27 \log _{10} \mathrm{CFU} \cdot \mathrm{g}^{-1}\right.$, range $-7.96-$ $5.25 \log _{10} \mathrm{CFU} \cdot \mathrm{g}^{-1} ; \mathrm{p}<0.001$ ) (fig. 2a). A nonsignificant reduction in CFU count in the ciprofloxacin DPI group compared with placebo was observed on days 42 and 56 before the plots joined again at day 84 . In the per-protocol analysis, a similar pattern of mean reduction in CFU count during treatment and at EOT was observed in the ciprofloxacin DPI group compared with the placebo group $(\mathrm{p}<0.001)$ (fig. $2 \mathrm{~b})$. Mean colony counts of selected pathogens at baseline and at EOT are presented in figure 3 .

\section{Secondary end-points}

Fewer subjects reported at least one exacerbation throughout the study in the ciprofloxacin DPI group (22 (36.7\%) out of 60
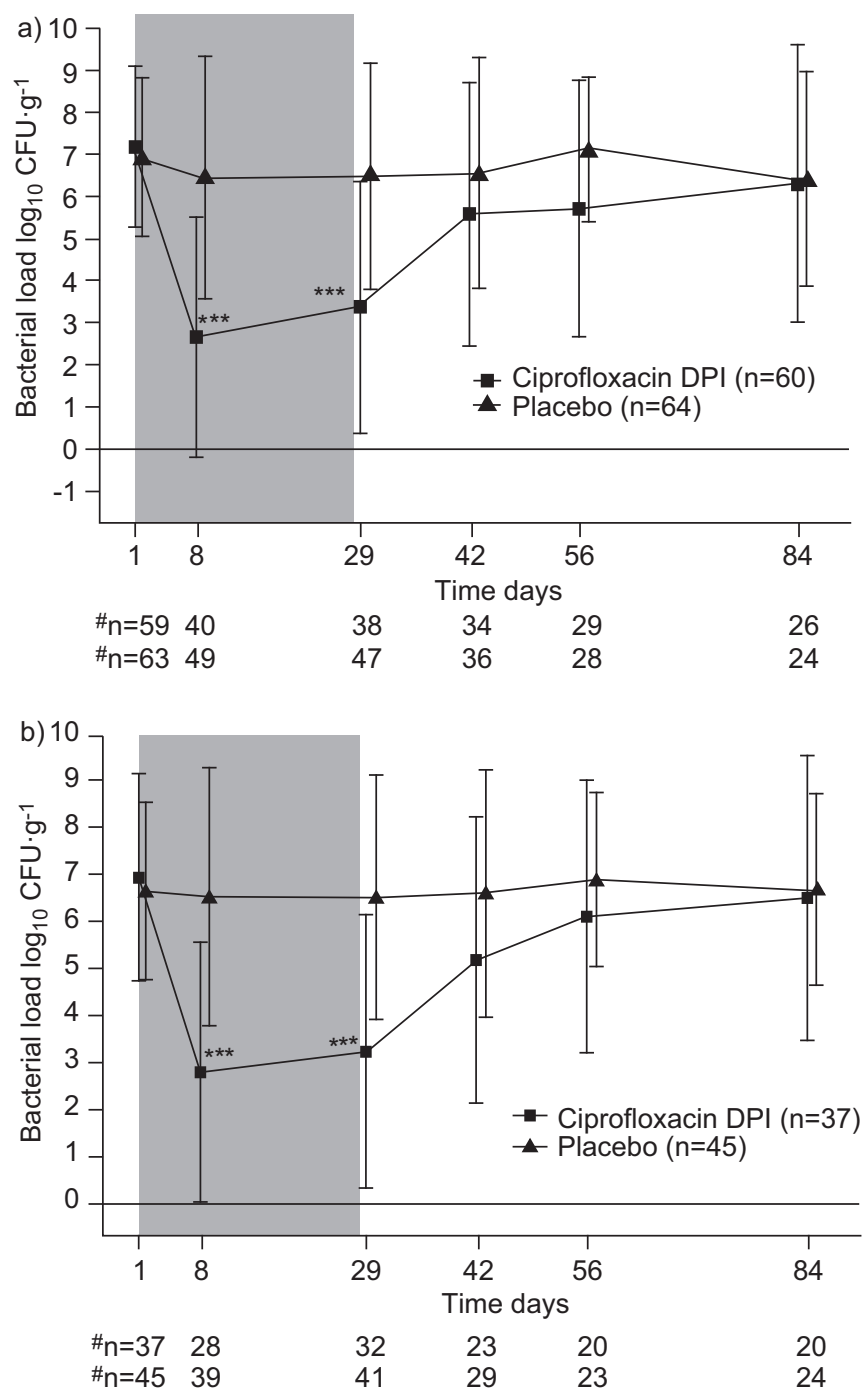

FIGURE 2. Mean bacterial load for the a) modified intent-to-treat population and b) per-protocol populations. Only results from valid cultures were considered. Cultures were excluded if subjects were administered concomitant antibacterials, if there were $>25$ squamous epithelial cells in the absence of Pseudomonas aeruginosa or if there were $\leqslant 25$ leukocytes for $P$. aeruginosa-negative cultures pretherapy. DPI: dry powder for inhalation. Shaded area indicates treatment period (days). ${ }^{*}$ : number of patients with valid sputum cultures. ${ }^{* *}$ : $p<0.001$.

subjects) than in the placebo group (25 (39.1\%) out of 64 subjects; hazard ratio estimate $0.802,95 \%$ CI $0.443-1.454$; $\mathrm{p}=0.605)$. The number of subjects with new-onset exacerbations requiring intervention per time period is presented in figure 4 . Of these, 14 subjects in the ciprofloxacin DPI group required intervention with antibacterial therapy, including two subjects who required hospitalisation, compared with 18 subjects in the placebo group who required antibacterial therapy, including five subjects who required hospitalisation (hazard ratio estimate 0.674, 95\% CI 0.324-1.402; $\mathrm{p}=0.338$ (stratified log-rank test, stratification by centre, small centres pooled)). All subjects with an exacerbation requiring antibacterial therapy should have been discontinued according to the study protocol; however, three of these subjects (two in the ciprofloxacin DPI group and one in the placebo group) 


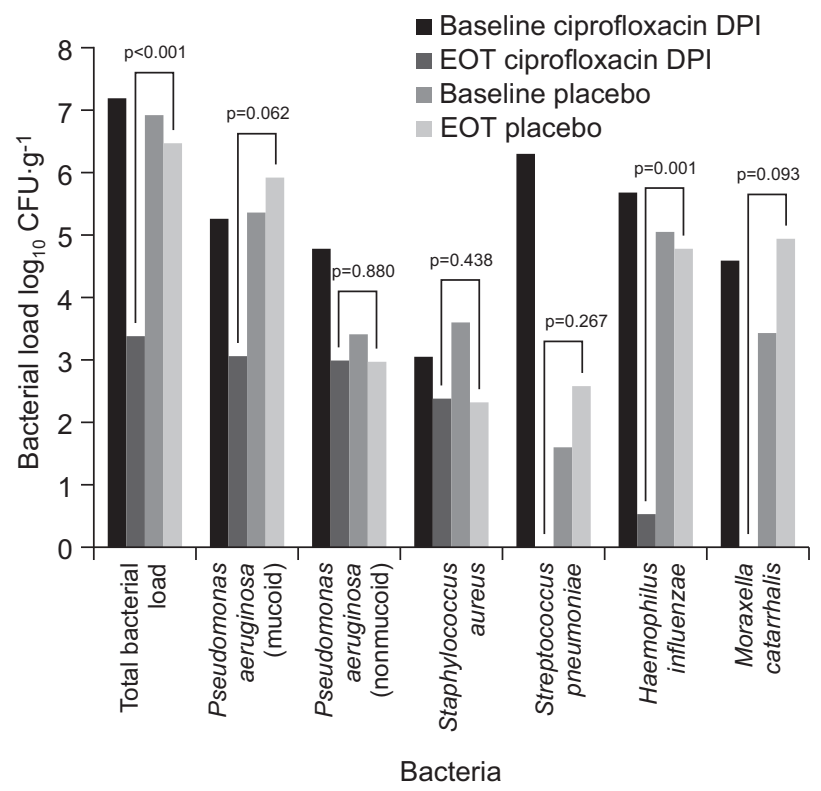

FIGURE 3. Mean colony count of selected pathogens at baseline and at end of treatment. Only results from valid cultures were considered. Cultures were excluded if subjects were administered concomitant antibacterials, if there were $>25$ squamous epithelial cells in absence of Pseudomonas aeruginosa or if there were $\leqslant 25$ leukocytes for $P$. aeruginosa-negative cultures pre-therapy. DPI: dry powder for inhalation; EOT: end of treatment.

completed the study. Further information on these three subjects is included in the online supplementary material. The number of exacerbations requiring antibacterial therapy in the first 42 days ( 28 days of treatment and 14 days of followup; a time period likely to be most influenced by the antibacterial treatment) was seven in the ciprofloxacin DPI group and 12 in the placebo group. The rate of exacerbations requiring intervention in subjects with and without P. aeruginosa at baseline was similar in the two groups $(23.5 \%$ in the ciprofloxacin DPI group and $27.8 \%$ in the placebo group versus $23.1 \%$ in the ciprofloxacin DPI group and $25.0 \%$ in the placebo group, respectively). An overview of exacerbations is presented in the online supplementary material.

Eradication (negative bacterial culture; only results from valid cultures were considered) was observed in $20(48 \%)$ out of 42 of subjects in the ciprofloxacin DPI group compared with six $(12 \%)$ out of 51 of those in the placebo group at day $8(\mathrm{p}<0.001)$, and in $14(35 \%)$ out of 40 subjects in the ciprofloxacin DPI and four $(8 \%)$ out of 49 of those in the placebo group at EOT $(p=0.001)$ (table 2). The emergence of a new potential respiratory pathogen during treatment was less frequent in subjects receiving ciprofloxacin DPI than in those in the placebo group (12 and 24 subjects, respectively). The new pathogens isolated post-baseline were similar in frequency to those isolated at baseline (table 1), except for S. maltophilia, which was isolated in five subjects at baseline (two on ciprofloxacin DPI and three on placebo) compared with 17 subjects post-baseline (10 on ciprofloxacin DPI and seven on placebo).

The total sputum volume recovered at EOT was less than that at baseline for both groups; mean percentage change from baseline was $-17.7 \%$ and $-9.6 \%$ for the ciprofloxacin DPI and

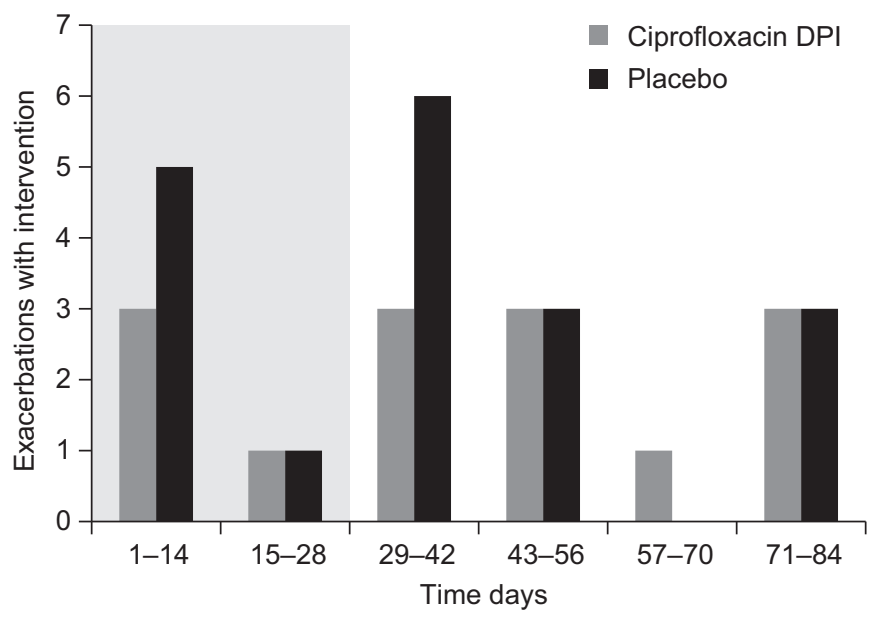

FIGURE 4. Occurrence of exacerbations requiring antibacterial intervention throughout the study (modified intent-to-treat population). 22 subjects in the ciprofloxacin DPI group and 25 subjects in the placebo group experienced an exacerbation. Of these, 14 subjects in the ciprofloxacin DPI group and 18 subjects in the placebo group required antibacterial treatment. DPI: dry powder for inhalation. Shaded area indicates treatment period (days).

placebo groups, respectively. More subjects had clear sputum colour at EOT and at the 4-week follow-up in the ciprofloxacin DPI group compared with the placebo group (table 3).

There was no observed effect on FEV1 or FVC in either the ciprofloxacin DPI or placebo groups. The mean \pm SD change in FEV1 from baseline to EOT for the ciprofloxacin DPI group was an improvement of $0.06 \pm 8.36 \%$ pred and a deterioration of $-0.40 \pm 10.06 \%$ pred for the placebo group.

Mean high-sensitivity C-reactive protein values between randomisation and EOT decreased in the ciprofloxacin DPI group but increased in the placebo group $\left(-1.09 \mathrm{mg} \cdot \mathrm{L}^{-1}\right.$ versus $4.02 \mathrm{mg} \cdot \mathrm{L}^{-1}$, respectively; $\mathrm{p}=0.173$ ). Evidence for a treatment effect on absolute neutrophil count was indicated by a significant reduction at EOT for ciprofloxacin DPI compared with placebo $\left(-0.36 \times 10^{6}\right.$ cells $\cdot \mathrm{mL}^{-1}$ versus $0.60 \times 10^{6}$ cells $\cdot \mathrm{mL}^{-1}$, respectively; $\mathrm{p}=0.014)$.

Increases in minimum inhibitory concentration (MIC) to $>4 \mathrm{mg} \cdot \mathrm{L}^{-1}$ (a level defined as clinically relevant for systemic therapy with ciprofloxacin), which were considered to be probably or possibly related to the administration of study drug, were reported up to the end of the study for six subjects in the ciprofloxacin DPI group and no subjects in the placebo group for the following pathogens: $P$. aeruginosa $(n=4)$, S. maltophilia $(\mathrm{n}=1)$ and $H$. influenzae $(\mathrm{n}=1)$. Of these subjects, one prematurely discontinued the study and four showed MIC decreases to susceptible levels by the end of the study. One subject with mucoid $P$. aeruginosa maintained resistance, which peaked at day 30 (MIC $64 \mathrm{mg} \cdot \mathrm{L}^{-1}$ ) and decreased to $4 \mathrm{mg} \cdot \mathrm{L}^{-1}$ (day 44 and day 63) and $8 \mathrm{mg} \cdot \mathrm{L}^{-1}$ at day 92 . One subject in the placebo group experienced a transient MIC increase in S. maltophilia at EOT from $1 \mathrm{mg} \cdot \mathrm{L}^{-1}$ to $4 \mathrm{mg} \cdot \mathrm{L}^{-1}$.

At EOT, the mean change from baseline in SGRQ score for subjects in the ciprofloxacin DPI and placebo groups was -2.52 points and +0.38 points, respectively, indicating an improvement in the 
TABLE 2 Number of subjects with eradication (negative bacterial culture, modified intent-to-treat population)

\begin{tabular}{lccc} 
Time period & Ciprofloxacin DPI & Placebo $^{\text {p-value }}$ \\
\hline During treatment visit (day 8) & $20 / 42(47.6)$ & $6 / 51(11.8)$ & $<0.001$ \\
End of treatment (day 29) & $14 / 40(35.0)$ & $4 / 49(8.2)$ & 0.001 \\
2-week follow-up (day 42) & $6 / 36(16.7)$ & $4 / 37(10.8)$ & 0.359 \\
4-week follow-up (day 56) & $4 / 31(12.9)$ & $1 / 28(3.6)$ & 0.617 \\
8-week follow-up (day 84) & $4 / 27(14.8)$ & $2 / 25(8.0)$ & 0.763 \\
\hline
\end{tabular}

Data are presented as n/N (\%), unless otherwise stated. DPI: dry powder for inhalation. ${ }^{\#}$ : based on Cochran-Mantel-Haenszel test stratified by centre/cluster of centre.

ciprofloxacin DPI group. The adjusted mean difference in SGRQ scores for ciprofloxacin DPI compared with placebo was $-3.56(95 \%$ CI -7.3-0.1; $\mathrm{p}=0.059)$; adjustment was made for baseline SGRQ and study centre. A similar trend was found in the responder analysis: $40 \%$ of subjects receiving ciprofloxacin DPI and $32 \%$ of subjects receiving placebo reported an improvement in score of more than four points at EOT.

\section{Safety}

A summary of treatment-emergent, drug-related and serious AEs is presented in table 4 . No deaths occurred and no serious AEs within this time period were considered to be related to study treatment. Discontinuations from the study are summarised in fig. 1.

Throughout the study (up to day 84), 22 subjects in the ciprofloxacin DPI group (three subjects prematurely discontinued treatment) and 26 subjects in the placebo group (six prematurely discontinued treatment) reported at least one $\mathrm{AE}$ of exacerbation of bronchiectasis. Of these, 18 events reported in 17 subjects in the ciprofloxacin DPI group and 14 events in 13 subjects in the placebo group were classified as "late events", occurring $>7$ days after EOT. A similar number of subjects prematurely discontinued the study because of AEs in each treatment group: $19(31.7 \%)$ subjects and $23(35.9 \%)$ subjects in ciprofloxacin DPI and placebo groups, respectively.

AEs of special interest in the ciprofloxacin DPI and placebo groups included bronchospasm (three out of 60 and three out of 64 subjects, respectively; two incidences in each group occurred during treatment and were considered to be treatment related). None of the investigator-reported AEs of bronchospasm (six in total) met the study-specific definition of bronchospasm. Treatment-emergent AEs of cough ( 0 out of 60 and five out of 64 subjects, respectively) and haemoptysis (one out of 60 and two out of 64 subjects, respectively) were reported.

No significant changes in laboratory parameters were recorded in either treatment group. Ciprofloxacin-induced changes in any liver function test or serum creatinine were not detected.

\section{DISCUSSION}

Relatively few randomised clinical trials have been conducted exclusively in subjects with non-CF bronchiectasis. Regular, long-term nebulised gentamicin was observed to be of significant benefit in subjects with non-CF bronchiectasis; however, continuous treatment was required to ensure ongoing efficacy [18]. At the 3-month follow-up, outcome measures were similar to baseline [18]. New therapies supported by a robust evidence base are needed.

This study was designed to include subjects who were chronically infected with a relevant pathogen and whose bronchiectasis was of sufficient severity to warrant considering treatment with inhaled antibiotics. We included non-CF subjects with post-infective and idiopathic bronchiectasis, who account for between $70 \%$ and $80 \%$ of all non-CF bronchiectasis cases $[19,20]$. We excluded aetiologies in which response to treatment could be affected by complicating factors, for example, poor mucus clearance attributable to primary ciliary dyskinesia, allergic inflammation and immune deficiency. A relatively large number of study centres were required for recruitment but the results were conclusive despite any variability this may have introduced, and the interaction term between treatment and centre for the main analysis was not significant $(p=0.214)$.

\begin{tabular}{lccc} 
TABLE 3 & $\begin{array}{c}\text { Sputum colour in the modified intent-to-treat/ } \\
\text { safety population with available sputum samples }\end{array}$ \\
& $\begin{array}{c}\text { Ciprofloxacin } \\
\text { DPI }\end{array}$ & Placebo & p-value \\
& & & \\
\hline & & & \\
Baseline total subjects & 60 & $63^{\#}$ & $0.220^{\circ}$ \\
No sputum/clear & $5(8.3)$ & $7(11.1)$ & \\
Yellow & $29(48.3)$ & $23(36.5)$ & \\
Green & $25(41.7)$ & $28(44.4)$ & \\
Rust & $1(1.7)$ & $5(7.9)$ & \\
End of treatment & 49 & 52 & $0.026^{\circ}, 0.029^{+}$ \\
No sputum/clear & $12(24.5)$ & $9(17.3)$ & \\
Yellow & $31(63.3)$ & $26(50.0)$ & \\
Green & $5(10.2)$ & $17(32.7)$ & \\
Rust & $1(2.0)$ & 0 & \\
4-week follow-up & 40 & 37 & $0.494^{\circ}, 0.609^{+}$ \\
No sputum/clear & $10(25.0)$ & $5(13.5)$ & \\
Yellow & $16(40.0)$ & $22(59.5)$ & \\
Green & $11(27.5)$ & $9(24.3)$ & \\
Rust & $3(7.5)$ & $1(2.7)$ & \\
\hline
\end{tabular}

Data are presented as $\mathrm{n}$ or $\mathrm{n}(\%)$, unless otherwise stated. DPI: dry powder for inhalation. ${ }^{*}$ : no sputum information was available for one patient; " : based on Cochran-Mantel-Haenszel test with ridit scores test stratified by centre/cluster of centre; $^{+}$: based on Cochran-Mantel-Haenszel test with ridit scores test stratified by centre/cluster of centre and baseline sputum colour. 
TABLE 4 Summary of adverse events (AEs) (modified intent-to-treat/safety population)

\begin{tabular}{|c|c|c|c|}
\hline AE & Ciprofloxacin DPI & Placebo & p-value \\
\hline Subjects $\mathbf{n}$ & 60 & 64 & \\
\hline Patients with any treatment-emergent AEs & $41(68.3)$ & $42(65.6)$ & 0.849 \\
\hline Patients with treatment-related AEs & $21(35.0)$ & $17(26.6)$ & 0.335 \\
\hline \multicolumn{4}{|l|}{ Treatment-emergent AEs reported in $>5 \%$ subjects } \\
\hline Cough & 0 & $5(7.8)$ & 0.058 \\
\hline Exacerbation of bronchiectasis & $7(11.7)$ & $14(21.9)$ & 0.155 \\
\hline Headache & $4(6.7)$ & $5(7.8)$ & 1.000 \\
\hline Subjects with treatment-emergent serious $\mathrm{AEs}^{\#}$ & $2(3.3)$ & $3(4.7)$ & 1.000 \\
\hline Complex pain syndrome & $1(1.7)$ & 0 & 0.484 \\
\hline
\end{tabular}

The results of the current study show, for the first time in nonCF bronchiectasis, that ciprofloxacin DPI produced a statistically significant reduction in total bacterial load after 28 days of treatment compared with placebo. The more than three-logfold reduction in bacterial counts is equivalent to a $>99.9 \%$ reduction in bacterial load. Moreover, a greater percentage of subjects receiving ciprofloxacin DPI had negative bacterial culture at EOT than did subjects receiving placebo. A reduction in colony count for the two most prevalent pathogens in nonCF bronchiectasis, $P$. aeruginosa and $H$. influenzae, was observed with ciprofloxacin DPI treatment. Although the study was not powered to show a significant difference in the number of subjects reporting exacerbations, a trend was observed favouring ciprofloxacin DPI, suggesting that it might be a useful agent in the management of this patient population. Reduction of bacterial load in the lungs should reduce both airway inflammation and the risk of exacerbations, which is supported by the observation of a reduction in sputum purulence. Prior data support associations between sputum colour and bronchial inflammation [21]. Hence, the results of this study show an emerging pattern of clinically meaningful effects on the pathophysiology of bronchiectasis.

The most common dosing interval used for the aerosolised antibacterials currently available or in development is a cycle of 28 days on treatment followed by 28 days off treatment. A rapid return to near baseline values in bacterial load was observed between EOT and the end of the study. However, at day 56, which would correspond to the return to treatment in this proposed cycled regimen, the decrease in bacterial burden was still apparent. The rapid drop in bacterial load followed by rebound is consistent with the apparent impact of ciprofloxacin DPI on the pattern of exacerbations, i.e. suppression relative to placebo in the first 42 days of the study and incidence rates becoming similar thereafter.

An important rationale for using this regimen (28 days on/ 28 days off treatment) is to minimise the development of antibacterial resistance. Resistance can develop after exposure of a pathogen to an antibacterial; the 28-day off-treatment period removes the selective pressure of the antibacterial, allowing pathogen susceptibility to return towards its pretreatment level $[22,23]$. Potential resistance development, as indicated by changes in MIC, was observed in this study: isolates from six subjects in the ciprofloxacin DPI group demonstrated MIC increases by EOT, and subsequent MIC decreases in isolates from four of these subjects were reported by day 56 .

In addition to improvements in clinical efficacy parameters, trends towards improvements in patient-reported health status for subjects receiving ciprofloxacin DPI were observed with the SGRQ at EOT, despite the brief treatment period. This was close to a clinically relevant improvement (-4.0 points in SGRQ; lower scores represent improved HRQoL) [24]. This trend was also observed at day 56 but not at the end of the study, indicating that any reported benefit on health status was temporary.

Ciprofloxacin DPI $32.5 \mathrm{mg}$ b.i.d. was well tolerated; the AEs observed are common in subjects with respiratory diseases, with the most frequently reported AE being exacerbations [25, 26]. No significant changes in laboratory parameters were observed and there was a low incidence of drug-related AEs, with no significant differences observed between the ciprofloxacin DPI and placebo groups. The incidence of AEs associated with non-CF bronchiectasis, including bronchospasm, cough and haemoptysis, was low throughout the treatment period.

Ciprofloxacin DPI $32.5 \mathrm{mg}$ achieved a significant reduction in bacterial load compared with placebo in subjects with postinfective or idiopathic non-CF bronchiectasis whose disease profile and severity warranted consideration of inhaled antibiotic therapy. Ciprofloxacin DPI was well tolerated when administered b.i.d. for 28 days. The improvements observed 
with ciprofloxacin DPI in secondary clinical and quality of liferelated end-points were not statistically significant. Further studies powered to evaluate the clinical effects of long-term intermittent ciprofloxacin DPI therapy are therefore needed in this patient population.

\section{SUPPORT STATEMENT}

The study was sponsored by Bayer Pharma AG, Germany. R. Wilson and A. De Soyza wish to acknowledge the support of the National Institute for Health Research (NIHR) infrastructure in the form of NIHR Biomedical Research Unit funding (Royal Brompton Hospital, London, UK) and the Comprehensive Local Research Networks funding (other UK centres).

\section{CLINICAL TRIAL}

This study is registered at www.clinicaltrials.gov with identifier number NCT00930982.

\section{STATEMENT OF INTEREST}

Conflict of interest information can be found alongside the online version of this article at www.erj.ersjournals.com

\section{ACKNOWLEDGEMENTS}

All analyses were carried out by the study sponsor and the Data Review Committee was responsible for validating all clinical failure data. The authors would also like to acknowledge D. Bilton (Royal Brompton Hospital, London, UK) and G. Tino (Hospital of the University of Pennsylvania, PA, USA), for their work on the blinded interim analysis, L. Zhou (third-party statistician; Axio Research Seattle, Washington, DC, USA) and F. Woodward (Chameleon Communications International, London, UK), who provided medical writing services with funding from Bayer Pharma AG. The authors wish to thank the Principal Investigators at each of the recruiting centres: Australia: John Armstrong (Princess Alexandra Hospital, Brisbane), Michael Chia (Burnside War Memorial Hospital, Toorak Gardens, Adelaide), Harvey Newnham (Alfred Hospital, Melbourne), Fergal O'Donoghue (Austin Hospital, Heidelberg, Melbourne), Matthew Peters (Concord Repatriation General Hospital, Concord, Sydney), David Serisier (Mater Health Services, South Brisbane), Philip Thompson (Sir Charles Gairdner Hospital, Nedlands, Perth); Germany: Helgo Magnussen (Pneumologisches Forschungsinstitut $\mathrm{GmbH}$ am Krankenhaus Großhansdorf, Großhansdorf), Stephanie Korn (Universitätsmedizin der Johann Gutenberg Universität Mainz, Mainz), Gerd Stähler (Klinik Löwenstein dGmbH, Löwenstein), Andreas Eich (Interdisziplinäres Facharztzentrum Sachsenhausen (IFS), Infektiologikum, Frankfurt), Olaf Burkhardt (Zentralklinik Bad Berka, Bad Berka), Lutz Volgmann (Pneumologische Praxis Hannover Nordstadt, Hannover), Karlheinz Franz (Lungenpraxis Witten, Witten), Axel Kroker (Pneumologische Schwerpunktpraxis Kroker, Schaeben, Schmidt, Koblenz), Ekkehard Beck (IFG Institut für Gesundheitsförderung $\mathrm{GmbH}$, Rüdersdorf), Andrés De Roux (Pneumologische Praxis am Schloß Charlottenburg, Berlin), Petra Mikloweit (Pneumologisches Forschungsinstitut Hohegeest GbR, Geesthacht), Lutz von Versen (Praxis Walter Meier, Berlin), Thomas Schultz (MECS GmbH, Berlin); Spain: Marcelino Salgueiro Rodriguez and Rosario Arias (Complejo Hospitalario Universitario de Santiago, Santiago de Compostela), Pilar Cordero (Hospital Universitario Infanta Cristina, Badajoz), Jaume Sauleda Roig (Hospital Universitario Son Dureta, Palma de Mallorca); Sweden: Annika Hollsing (Uppsala CF Center, Uppsala); UK: Joseph Elborn (Belfast City Hospital, Belfast), Charles Haworth (Papworth Hospital, Cambridge), Nabil Jarad (Bristol Royal Infirmary Hospital, Bristol), Paul Walker (University Hospital Aintree, Liverpool), Andrew Wilson (Norfolk and Norwich University Hospital, Norwich); and USA: Tala'at Al-Shuquairat (Central Utah Clinic, Provo, UT), Mark Metersky (University of Connecticut, Farmington, CT), Varsha Taskar (University of Texas Health, Tyler,
TX), Angela Wang (Scripps Clinical Medical Group, La Jolla, CA), Jonathan Ilowite (Winthrop University Hospital, Mineola, NY), Jose Marquina (Pulmonary, Critical Care and Sleep Medicine, Naples, FL), Gwen Huitt (National Jewish Health, Denver, CO) and Ikeadi Ndukwu (La Porte County Institute for Clinical Research, Inc., Michigan City, IN).

\section{REFERENCES}

1 Seitz AE, Olivier KN, Adjemian J, et al. Trends in bronchiectasis among Medicare beneficiaries in the United States, 2000-2007. Chest 2012; 142: 432-439.

2 Loebinger MR, Wells AU, Hansell DM, et al. Mortality in bronchiectasis: a long-term study assessing the factors influencing survival. Eur Respir J 2009; 34: 843-849.

3 Angrill J, Agusti C, de Celis R, et al. Bacterial colonisation in patients with bronchiectasis: microbiological pattern and risk factors. Thorax 2002; 57: 15-19.

4 Smith MP. Non-cystic fibrosis bronchiectasis. J R Coll Physicians Edinb 2011; 41: 132-139.

5 King PT. The pathophysiology of bronchiectasis. Int J Chron Obstruct Pulmon Dis 2009; 4: 411-419.

6 O'Donnell AE. Bronchiectasis. Chest 2008; 134: 815-823.

7 Hill AT, Pasteur M, Cornford C, et al. Primary care summary of the British Thoracic Society Guideline on the management of noncystic fibrosis bronchiectasis. Prim Care Respir J 2011; 20: 135-140.

8 Rubin BK. Aerosolized antibiotics for non-cystic fibrosis bronchiectasis. J Aerosol Med Pulm Drug Deliv 2008; 21: 71-76.

9 Woodhead M. New guidelines for the management of adult lower respiratory tract infections. Eur Respir J 2011; 38: 1250-1251.

10 Chalkley LJ, Koornhof HJ. Antimicrobial activity of ciprofloxacin against Pseudomonas aeruginosa, Escherichia coli, and Staphylococcus aureus determined by the killing curve method: antibiotic comparisons and synergistic interactions. Antimicrob Agents Chemother 1985; 28: 331-342.

11 Gupta A, Tarara T, Miller D, et al. A novel high-payload dry powder formulation of ciprofloxacin for management of chronic lung infections. Am Assoc Pharmaceut Scientists J 2011; 13(S2): abstract R6156.

12 Stass H, Baumann-Noss S, Delesen H, et al. Ciprofloxacin PulmoSphere ${ }^{\circledR}$ inhalational powder: a healthy volunteer study. Am J Respir Crit Care Med 2008; 177: G50.

13 Stass H, Ludwig M, Nagelschmitz J, et al. Safety and pharmacokinetics of inhaled dry powder ciprofloxacin after single and multiple inhalations in adult patients with cystic fibrosis. Paediatr Pulmonol 2008; 43(S31): abstract 282, p. 300.

14 Tokimatsu I, Hiramatsu K, Morimoto T, et al. Safety, tolerability and pharmacokinetics of ciprofloxacin dry powder for inhalation in patients with mild to moderate chronic obstructive pulmonary disease: a randomized controlled trial. Am J Respir Crit Care Med 2011; 183: A3105.

15 Wilson $\mathrm{CB}$, Jones PW, O'Leary CJ, et al. Validation of the St. George's Respiratory Questionnaire in bronchiectasis. Am J Respir Crit Care Med 1997; 156: 536-541.

16 Jones PW, Quirk FH, Baveystock CM, et al. A self-complete measure of health status for chronic airflow limitation. The St. George's Respiratory Questionnaire. Am Rev Respir Dis 1992; 145: 1321-1327.

17 Hester KL, Macfarlane JG, Tedd H, et al. Fatigue in bronchiectasis. QJM 2012; 105: 235-240.

18 Murray MP, Govan JR, Doherty CJ, et al. A randomized controlled trial of nebulized gentamicin in non-cystic fibrosis bronchiectasis. Am I Respir Crit Care Med 2011; 183: 491-499.

19 Pasteur MC, Helliwell SM, Houghton SJ, et al. An investigation into causative factors in patients with bronchiectasis. Am J Respir Crit Care Med 2000; 162: 1277-1284. 
20 Shoemark A, Ozerovitch L, Wilson R. Aetiology in adult patients with bronchiectasis. Respir Med 2007; 101: 1163-1170.

21 Stockley RA, Bayley D, Hill SL, et al. Assessment of airway neutrophils by sputum colour: correlation with airways inflammation. Thorax 2001; 56: 366-372.

22 Fiel SB. Aerosolized antibiotics in cystic fibrosis: current and future trends. Expert Rev Respir Med 2008; 2: 479-487.

23 LiPuma JJ. Microbiological and immunologic considerations with aerosolized drug delivery. Chest 2001; 120: 118S-123S.
24 Jones PW. St. George's Respiratory Questionnaire: MCID. COPD 2005; 2: 75-79.

25 Martinez-Garcia MA, Soler-Cataluna JJ, Perpina-Tordera M, et al. Factors associated with lung function decline in adult patients with stable non-cystic fibrosis bronchiectasis. Chest 2007; 132: 1565-1572.

26 Ryan G, Singh M, Dwan K. Inhaled antibiotics for long-term therapy in cystic fibrosis. Cochrane Database Syst Rev 2011; 3: CD001021. 